\title{
MAMMALS
}

\section{SASKATCHEWAN CHRISTMAS MAMMAL COUNT - 1998}

Compiled by Wayne C. Harris, Saskatchewan Environment and Resource Management, 350 Cheadle Street West, Swift Current, SK S9H 4G3

The number of mammal counts conducted this year was 87 . Although fewer than last years record, it is still the second highest number ever. The number of species recorded was 39 , the same as last year. The average number of species per count (7.2), which is higher than last year but still below the long-term average.

Weather was warmer than usual up to the count period and then normal temperatures prevailed. The lack of snow for most localities during the count meant that animals remained dispersed and tracks were hard to find; only those counts conducted later in the count period had good tracking snow. No new species were reported. The only species found this year which are irregularly reported were Woodland Caribou and Mountain Lion. Ironically the Woodland Caribou was at Candle Lake, the same count that last reported this species in the 1970's.

Mountain Lion tracks were found Robert Wapple and Wayne Renaud in Coal Mine Coulee on the Herschal count. Robert writes that "Fresh snow the previous night resulted in very distinct tracks. The tracks went in both directions, indicating that the lion had travelled along the creek and returned via the same route. Individual paw prints were estimated to be the size of my fist (we had no rule). Distinct claw marks were evident on some of the tracks imprinted in the skiffs of fresh snow on the wind-polished creek ice, indicating that the lion needed some extra traction on the slippery ice. Unfortunately, we didn't try to distinguish between front and back paws. I tried to approximate the distances between print sets and found that stride distance was approximately one normal pace, which would be .5 to $.75 \mathrm{~m}$. No tail marks were observed in the snow between the tracks."

White-tailed Deer were the most frequently encountered species with $84 \%$ of counts recording them. Coyotes were next at $78 \%$ followed by Red Fox $(54 \%)$ and Snowshoe Hare (53\%). White-tailed Jackrabbit (44\%) were less frequent than usual but this may be an artifact of poor tracking conditions.

For weather, coverage and participants please refer to the Christmas Bird Count found elsewhere in this issue. In the tables, a numeral alone shows that the mammals were seen, while a letter following the number means that the number was inferred by the means defined below. A letter alone means that the species was present but estimating the numbers was impossible or that no attempt was made to do so. 
Symbols used in the tables are:

$\mathrm{T}=$ tracks

$L=$ active lodge or hut

$\mathrm{D}=$ dead animal found

$\mathrm{d}=$ fresh diggings found

$\mathrm{S}=$ smell or odour

$\mathrm{H}=$ heard

$+=$ present during the count period (December 18 to January 3 ) but not found on count day.

The tables are started on following page.

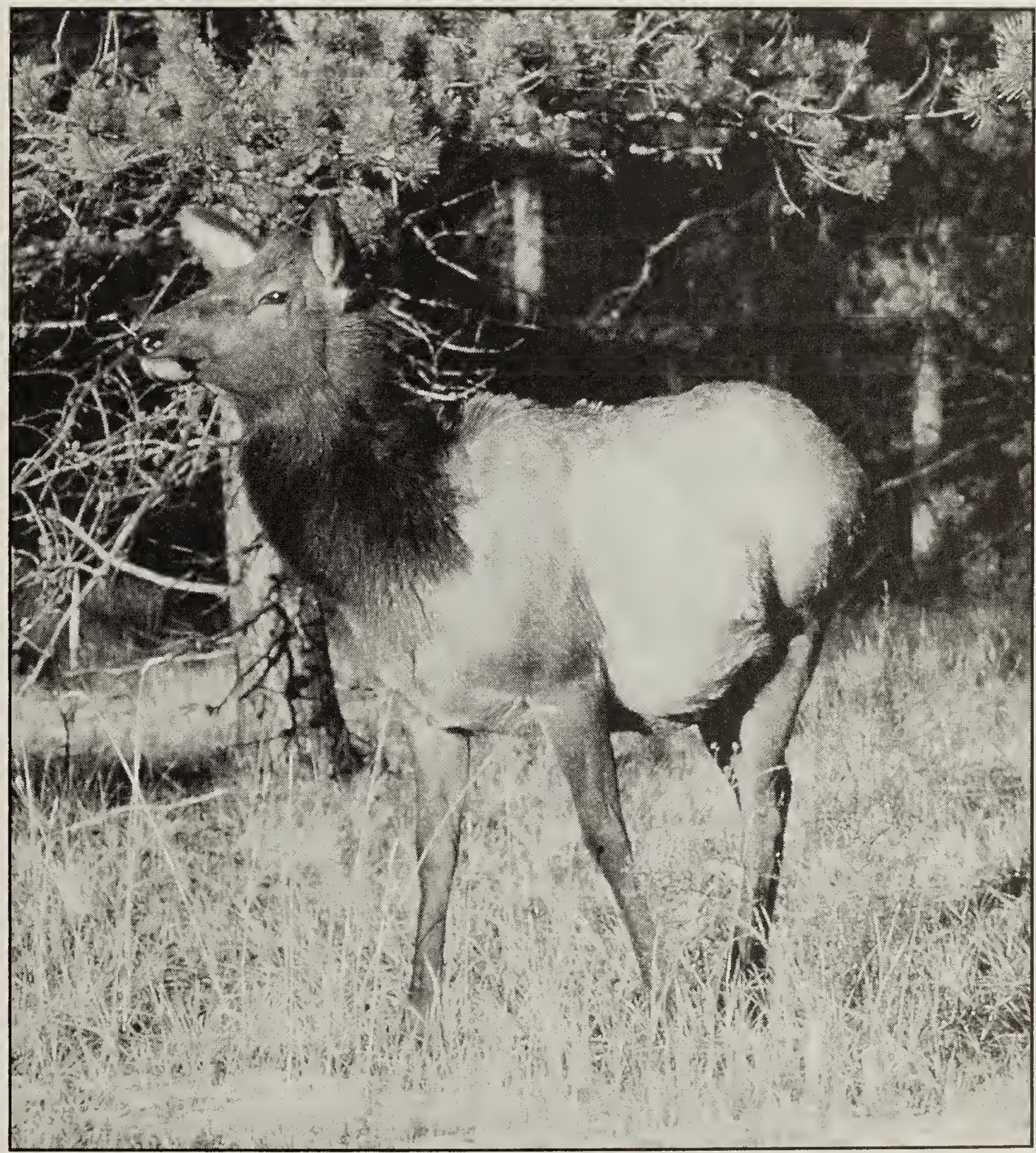


Table 1-1: SASKATCHEWAN CHRISTMAS MAMMAL COUNTS

SPECIES
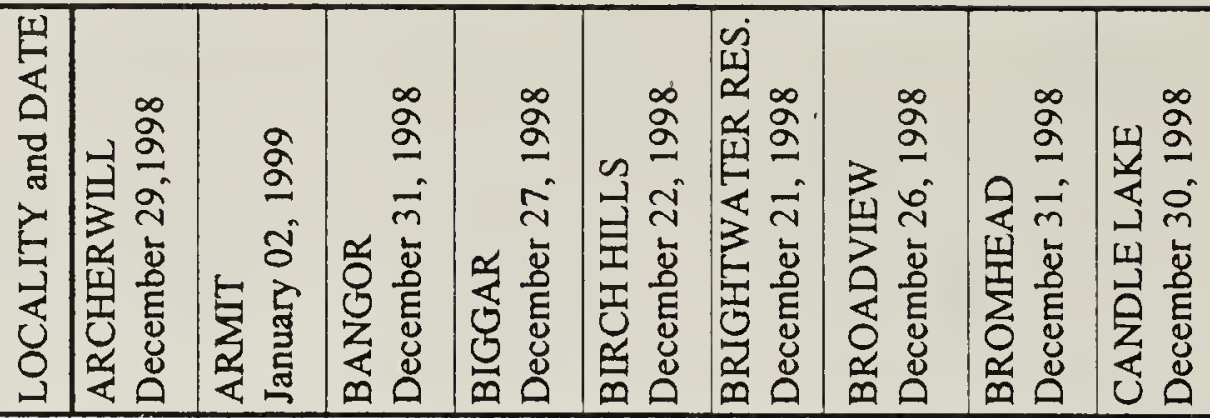

MASKED SHREW

SHREW species

EASTERN COTTONTAIL

NUTTALL'S COTTONTAIL

SNOWSHOE HARE

WHITE-TAILED JACKRABBIT

GREY SQUIRREL

FOX SQUIRREL

RED SQUIRREL

NORTHERN FLYING SQUIRREL

BEAVER

DEER MOUSE

GAPPER'S RED-BACKED VOLE

MUSKRAT

MEADOW VOLE

VOLE species

MOUSE species

NORWAY RAT

HOUSE MOUSE

PORCUPINE

COYOTE

WOLF

RED FOX

SWIFT FOX

BLACK BEAR

RACOON

FISHER

ERMINE

LONG-TAILED WEASEL

LEAST WEASEL

WEASEL species

MINK

BADGER

STRIPED SKUNK

RIVER OTTER

MOUNTAIN LION

WOODLAND CARIBOU

MULE DEER

WHITE-TAILED DEER

DEER species

MOOSE

ELK

PRONGHORN

\begin{tabular}{|c|c|c|c|c|c|c|c|c|}
\hline & & & & & & $2 T$ & & \\
\hline & & & & & & & & \\
\hline & & & & & & & & \\
\hline & $25 \mathrm{~T}$ & 2 & $6 \mathrm{~T}$ & $1+28 \mathrm{~T}$ & & $28 \mathrm{~T}$ & & $40 \mathrm{~T}$ \\
\hline 2 & & & $3 \mathrm{~T}$ & & & & $\mathrm{~T}$ & \\
\hline \multicolumn{9}{|l|}{4} \\
\hline & & & & & & 1 & & \\
\hline & 6 & 1 & & 2 & & $4+4 \mathrm{~T}$ & & $16 \mathrm{~T}$ \\
\hline & & & & & & & 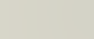 & \\
\hline \multicolumn{9}{|c|}{$2 \mathrm{~L}$} \\
\hline & & & & & & $4 \mathrm{~T}$ & & \\
\hline & & & & & & & & \\
\hline & $2 \mathrm{~L}$ & & $5 \mathrm{~L}$ & & & & & \\
\hline & & & & & & & & \\
\hline & & & & $3 \mathrm{~T}$ & & & & $2 \mathrm{~T}$ \\
\hline & $10 \mathrm{~T}$ & & $8 \mathrm{~T}$ & $7 \mathrm{~T}$ & & & & $3 T$ \\
\hline & & & & & & & is & \\
\hline \multirow[t]{2}{*}{$\mathrm{T}$} & & $2 \mathrm{~T}$ & & & & & & \\
\hline & & $2 \mathrm{~T}$ & & & & & & $1 \mathrm{~T}$ \\
\hline $2+4 \mathrm{~T}$ & $1+1 \mathrm{D}$ & $5 \mathrm{H}$ & $2+10 \mathrm{~T}$ & $2+6 \mathrm{~T}$ & 4 & $3 T$ & 1 & $3 \mathrm{~T}$ \\
\hline 1 & $13 \mathrm{~T}$ & & & & & & & \\
\hline \multirow[t]{8}{*}{1} & & 1 & 1 & & & $1 \mathrm{~T}$ & 2 & $1+1 \mathrm{~T}$ \\
\hline & & & & & & & & \\
\hline & & & & & & & & \\
\hline & & & & & & & & . \\
\hline & $1 \mathrm{~T}$ & & & & & & & IT \\
\hline & & & & $2 \mathrm{~T}$ & & $1 \mathrm{~T}$ & & \\
\hline & & & & & & $3 \mathrm{~T}$ & & $2 \mathrm{~T}$ \\
\hline & & $1 \mathrm{~T}$ & & & & & & \\
\hline \multirow[t]{8}{*}{$1+1 \mathrm{~T}$} & $1 \mathrm{~T}$ & & & & & & & \\
\hline & $1 \mathrm{~T}$ & & & $2 \mathrm{~T}$ & & $1 \mathrm{~T}$ & & $1 \mathrm{~T}$ \\
\hline & & & & & & & & \\
\hline & & & & & & & & \\
\hline & & & & & & & & IT \\
\hline & & & & & & & & \\
\hline & & & & & & & & $3 \mathrm{~T}$ \\
\hline & & & 38 & & 2 & 2 & & \\
\hline \multirow[t]{2}{*}{$2+7 \mathrm{~T}$} & 6 & 5 & 21 & & & $12+19 \mathrm{~T}$ & 51 & $5+39 \mathrm{~T}$ \\
\hline & $10 \mathrm{~T}$ & & & & & & & \\
\hline $5 \mathrm{~T}$ & $1 \mathrm{~T}$ & & & & & & & $4 T$ \\
\hline $5 \mathrm{~T}$ & $10 \mathrm{~T}$ & & & & & & & $8 \mathrm{~T}$ \\
\hline 11 & 14 & 8 & 8 & 7 & 2 & 12 & 5 & 15 \\
\hline
\end{tabular}


Table 1-2: SASKATCHEWAN CHRISTMAS MAMMAL COUNTS

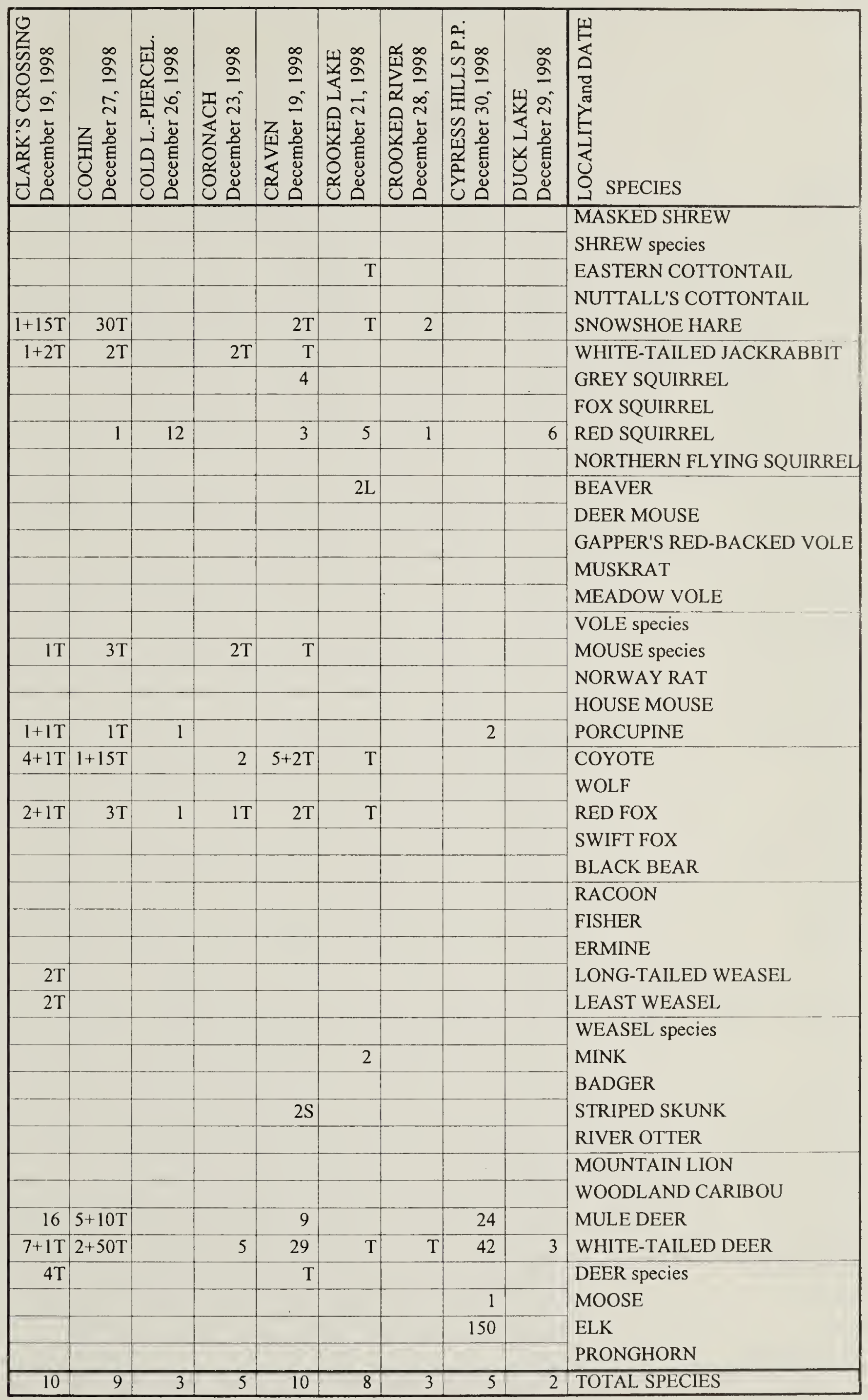


Table 1-3: SASKATCHEWAN CHRISTMAS MAMMAL COUNTS

\begin{tabular}{|c|c|c|c|c|c|c|c|c|c|c|}
\hline 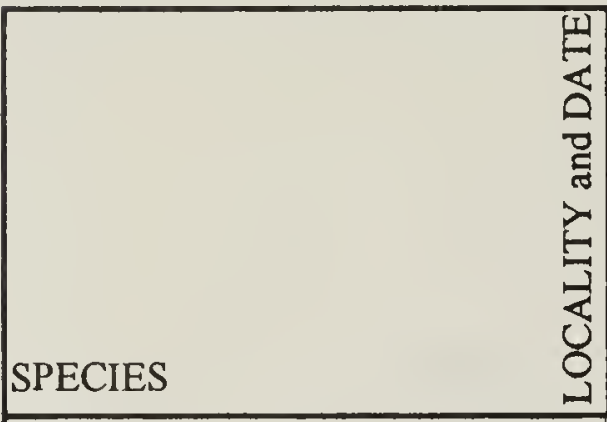 & 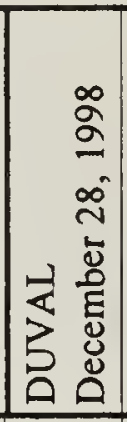 & 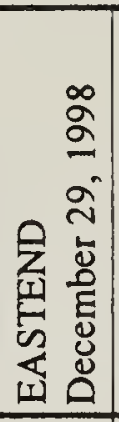 & 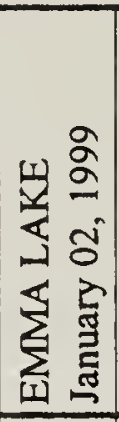 & 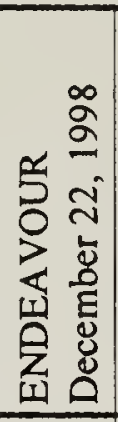 & 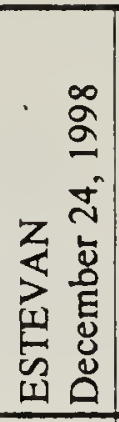 & 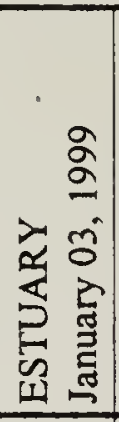 & 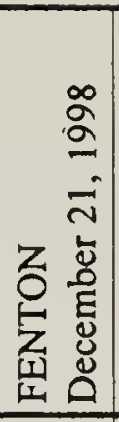 & 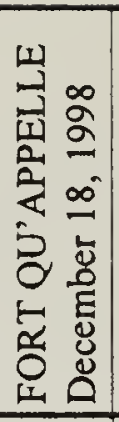 & 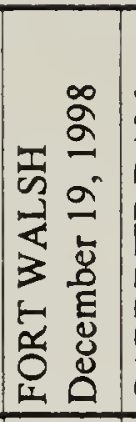 & 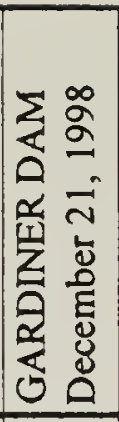 \\
\hline \multicolumn{11}{|l|}{ MASKED SHREW } \\
\hline \multicolumn{11}{|l|}{ SHREW species } \\
\hline \multirow{3}{*}{$\begin{array}{l}\text { EASTERN COTTONTAIL } \\
\text { NUTTALL'S COTTONTAIL } \\
\text { SNOWSHOE HARE }\end{array}$} & & & & & $4+10 \mathrm{~T}$ & & & & & \\
\hline & 11 & & & & & 2 & & & $5 \mathrm{~T}$ & $1+3 T$ \\
\hline & 2 & & $\mathrm{~T}$ & & & & $11 \mathrm{~T}$ & 11 & & $7 \mathrm{~T}$ \\
\hline \multirow{5}{*}{$\begin{array}{l}\text { WHITE-TAILED JACKRABBIT } \\
\text { GREY SQUIRREL } \\
\text { FOX SQUIRREL } \\
\text { RED SQUIRREL } \\
\text { NORTHERN FLYING SQUIRREL }\end{array}$} & 7 & & & & & & & 1 & & $4 \mathrm{~T}$ \\
\hline & & & & & & & & & & \\
\hline & & & & & $3+2 \mathrm{~T}$ & & & & & \\
\hline & & & 8 & + & & & $3+5 \mathrm{~T}$ & 6 & 7 & \\
\hline & & & & + & & & & & & \\
\hline \multirow{4}{*}{$\begin{array}{l}\text { BEAVER } \\
\text { DEER MOUSE } \\
\text { GAPPER'S RED-BACKED VOLE } \\
\text { MUSKRAT }\end{array}$} & $1 \mathrm{~L}$ & & & & & & & & $1 \%$ & \\
\hline & & & & & $2 \mathrm{~T}$ & & $4 \mathrm{~T}$ & + & $2 \mathrm{~T}$ & $1 \mathrm{~T}$ \\
\hline & & & & & & & & & & \\
\hline & $8 \mathrm{~L}$ & & & & & & & + & & \\
\hline MEADOW VOLE & & & & & & & & 1 & & \\
\hline \multicolumn{11}{|l|}{ VOLE species } \\
\hline MOUSE species & $3 \mathrm{~T}$ & & & & $5 \mathrm{~T}$ & & $2 \mathrm{~T}$ & & & \\
\hline \multicolumn{11}{|l|}{ NORWAY RAT } \\
\hline \multirow{2}{*}{$\begin{array}{l}\text { HOUSE MOUSE } \\
\text { PORCUPINE }\end{array}$} & & & & & & & & 1 & & \\
\hline & $2 \mathrm{~T}$ & $\mathrm{~T}$ & & 1T & 3 & 2 & & + & 1 & $6 \mathrm{~T}$ \\
\hline \multirow{2}{*}{$\begin{array}{l}\text { COYOTE } \\
\text { WOLF }\end{array}$} & $14 \mathrm{~T}$ & 1 & & iT & $2 \mathrm{~T}$ & 6 & $6 \mathrm{~T}$ & 1 & $1+5 \mathrm{~T}$ & $5+2 \mathrm{~T}$ \\
\hline & & & & + & & & & + & & \\
\hline RED FOX & $4 \mathrm{~T}$ & & & & $1 \mathrm{~T}$ & & & & $1 \mathrm{~T}$ & \\
\hline \multicolumn{11}{|l|}{ SWIFT FOX } \\
\hline \multicolumn{11}{|l|}{ BLACK BEAR } \\
\hline \multicolumn{11}{|l|}{ RACOON } \\
\hline \multicolumn{11}{|l|}{ FISHER } \\
\hline \multicolumn{11}{|l|}{ ERMINE } \\
\hline \multirow{2}{*}{$\begin{array}{l}\text { LONG-TAILED WEASEL } \\
\text { LEAST WEASEL }\end{array}$} & $2 \mathrm{~T}$ & & & & & & $1 \mathrm{~T}$ & + & & \\
\hline & & & & & & & $1 \mathrm{~T}$ & & $1 \mathrm{~T}$ & \\
\hline $\begin{array}{l}\text { WEASEL species } \\
\text { MINK }\end{array}$ & & & & & $1 \mathrm{~T}$ & & & & & \\
\hline MINK & & & 1 & & & & & + & & \\
\hline \multicolumn{11}{|l|}{ BADGER } \\
\hline \multicolumn{11}{|l|}{ STRIPED SKUNK } \\
\hline \multicolumn{11}{|l|}{ RIV SR OTTER } \\
\hline \multicolumn{11}{|l|}{ MOUNTAIN LION } \\
\hline WOODLAND CARIBOU & & & & & & & & & & \\
\hline MULE DEER & 3 & 3 & & & & 4 & & + & 15 & 116 \\
\hline WHITE-TAILED DEER & & 7 & $\mathrm{~T}$ & 6 & 20 & 22 & $4 \mathrm{~T}$ & + & 56 & 212 \\
\hline DEER species & $42 \mathrm{~T}$ & & & & & & & & & \\
\hline MOOSE & & & & $1 \mathrm{~T}$ & & & & & & \\
\hline ELK & & & & $1 \mathrm{~T}$ & & & & & $1+10 \mathrm{~T}$ & \\
\hline PRONGHORN & & & & & & & & + & 18 & \\
\hline TOTAL SPECIES & 11 & 4 & 4 & 8 & 8 & 5 & 7 & 15 & 12 & \\
\hline
\end{tabular}


Table 1-4: SASKATCHEWAN CHRISTMAS MAMMAL COUNTS

\begin{tabular}{|c|c|c|c|c|c|c|c|c|c|c|}
\hline 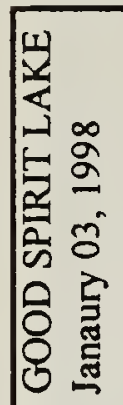 & 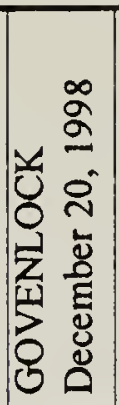 & 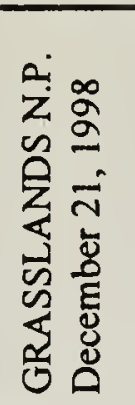 & 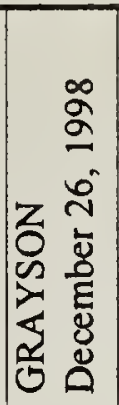 & 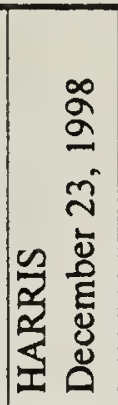 & 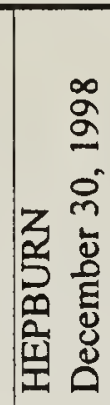 & 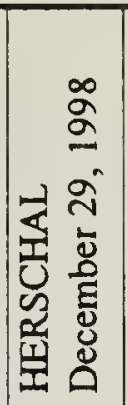 & 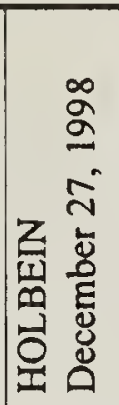 & 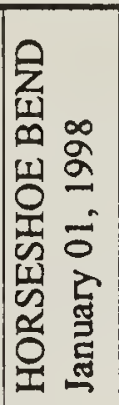 & 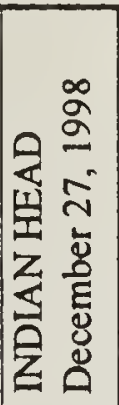 & SPECIES \\
\hline & & & & & & & & & & MASKED SHREW \\
\hline & & & & & & & & & & SHREW species \\
\hline & & & & & & & & & & EASTERN COTTONTAIL \\
\hline & $2+4 \mathrm{~T}$ & $1 \mathrm{~T}$ & & & & $2 \mathrm{~T}$ & & & & NUTTALL'S COTTONTAIL \\
\hline $10 \mathrm{~T}$ & & & & $6 \mathrm{~T}$ & & & & & 2 & SNOWSHOE HARE \\
\hline $8 \mathrm{~T}$ & & $4 \mathrm{~T}$ & $1 \mathrm{~T}$ & $1 \mathrm{~T}$ & & & & & & WHITE-TAILED JACKRABBIT \\
\hline & & & & & & & & & & GREY SQUIRREL \\
\hline & & & & & & & & & & FOX SQUIRREL \\
\hline 4 & & & & & 1 & & & 4 & 11 & RED SQUIRREL \\
\hline & & & & & & & & & & NORTHERN FLYING SQUIRREL \\
\hline & & & & & & & & & & BEAVER \\
\hline & & & & & & & & $6 \mathrm{~T}$ & & DEER MOUSE \\
\hline & & & & & & & & & & GAPPER'S RED-BACKED VOLE \\
\hline & & & & & & & & & & MUSKRAT \\
\hline & & & 2 & & & & & + & & MEADOW VOLE \\
\hline & & & & $8 \mathrm{~T}$ & & & & & & VOLE species \\
\hline & & & & & & $1 \mathrm{~T}$ & 2 & & & MOUSE species \\
\hline & & & & & & & & & & NORWAY RAT \\
\hline & & & & & & & & 1 & & HOUSE MOUSE \\
\hline 1 & & & & & & 1 & & & 2 & PORCUPINE \\
\hline $9 \mathrm{~T}$ & $2+1 \mathrm{~T}$ & $7+4 \mathrm{~T}$ & 1 & 2 & & $5+9 \mathrm{~T}$ & & 6 & 7 & COYOTE \\
\hline & & & & & & & & & & WOLF \\
\hline $2 \mathrm{~T}$ & & & & $4 \mathrm{~T}$ & & $4 \mathrm{~T}$ & & 3 & 1 & RED FOX \\
\hline & $2 \mathrm{~T}$ & & & & & & & & & SWIFT FOX \\
\hline & & & & & & & & & & BLACK BEAR \\
\hline & & & & & & & & & & RACOON \\
\hline & & & & & & & & & & FISHER \\
\hline & & & & & & & & & & ERMINE \\
\hline & & & & & & & & & & LONG-TAILED WEASEL \\
\hline & & $1 \mathrm{~T}$ & & & & & & 1 & & LEAST WEASEL \\
\hline $2 \mathrm{~T}$ & & & & & & $1 \mathrm{~T}$ & & & & WEASEL species \\
\hline & & & & & & & & & & MINK \\
\hline & & & & & & & & & & BADGER \\
\hline & & & & & & & & & & STRIPED SKUNK \\
\hline & & & & & & & & & & RIVEQ OTTER \\
\hline & & & & & & $1 \mathrm{~T}$ & & & & MOUNTAIN LION \\
\hline & & & & & & & & & & WOODLAND CARIBOU \\
\hline & 76 & 26 & & 48 & & 4 & & & & MULE DEER \\
\hline $76 \mathrm{~T}$ & 18 & & 17 & 16 & & 11 & & & 3 & WHITE-TAILED DEER \\
\hline & & $32+15 \mathrm{~T}$ & & & & & & & & DEER species \\
\hline $3 \mathrm{~T}$ & & & & & & $2 \mathrm{~T}$ & & & & MOOSE \\
\hline & & & & & & & & & & ELK \\
\hline & 165 & 234 & & & & 27 & & & & PRONGHORN \\
\hline 9 & 6 & 7 & 4 & 7 & $\overline{1}$ & 11 & & 7 & 6 & TOTAL SPECIES \\
\hline
\end{tabular}


Table 1-5: SASKATCHEWAN CHRISTMAS MAMMAL COUNTS

\begin{tabular}{|c|c|c|c|c|c|c|c|c|c|c|}
\hline 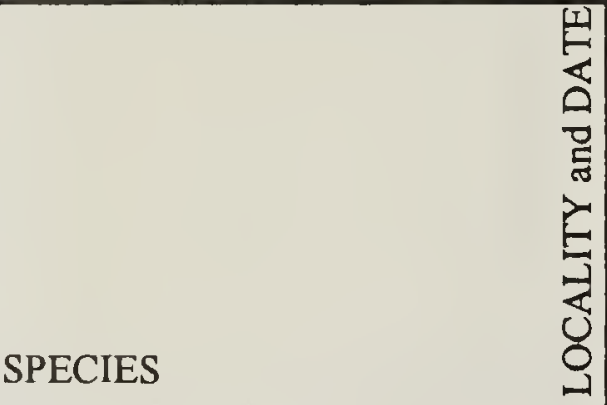 & 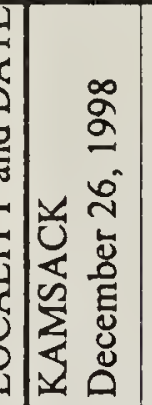 & 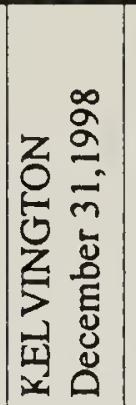 & 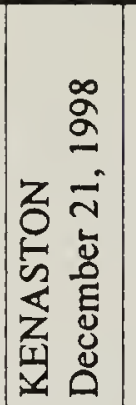 & 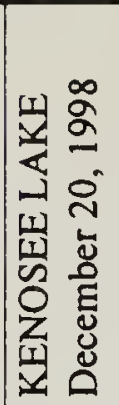 & 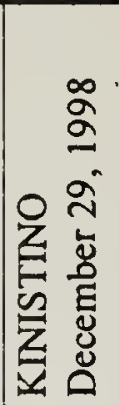 & 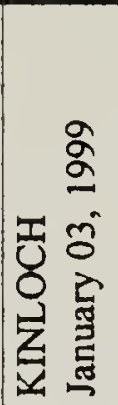 & 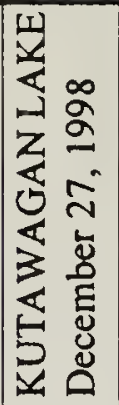 & 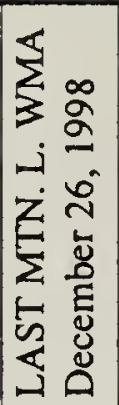 & 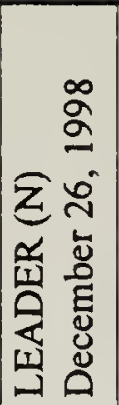 & 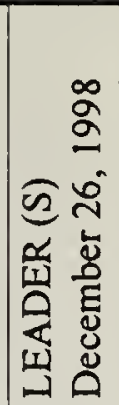 \\
\hline \multirow{5}{*}{$\begin{array}{l}\text { MASKED SHREW } \\
\text { SHREW species } \\
\text { EASTERN COTTONTAIL } \\
\text { NUTTALL'S COTTONTAIL } \\
\text { SNOWSHOE HARE }\end{array}$} & & & & & & & & & & \\
\hline & & & & & & & & & & \\
\hline & & & & $1 \mathrm{~T}$ & & & & & & \\
\hline & & & $\mathrm{T}$ & & & & & & 2 & \\
\hline & $\mathrm{T}$ & $21 \mathrm{~T}$ & & $1 \mathrm{~T}$ & & $30 \mathrm{~T}$ & & & & \\
\hline \multirow{5}{*}{$\begin{array}{l}\text { WHITE-TAILED JACKRABBIT } \\
\text { GREY SQUIRREL } \\
\text { FOX SQUIRREL } \\
\text { RED SQUIRREL } \\
\text { NORTHERN FLYING SQUIRREL }\end{array}$} & & $3 \mathrm{~T}$ & $5 \mathrm{~T}$ & & & & $2 \mathrm{~T}$ & $4 \mathrm{~T}$ & 2 & \\
\hline & & & & & & & & & & \\
\hline & & & & & & & & & & \\
\hline & & & & 5 & 1 & 4 & & & & \\
\hline & 2 & & & & & & & & & \\
\hline \multirow{5}{*}{$\begin{array}{l}\text { BEAVER } \\
\text { DEER MOUSE } \\
\text { GAPPER'S RED-BACKED VOLE } \\
\text { MUSKRAT } \\
\text { MEADOW VOLE }\end{array}$} & & & & $5 \mathrm{~L}$ & & $3 \mathrm{~L}$ & & & & \\
\hline & & & $\mathrm{T}$ & & & & & $1 \mathrm{~T}$ & 1 & \\
\hline & & & & & & & & & & \\
\hline & & & & $1 \mathrm{~L}$ & & & & & & \\
\hline & & & $\mathrm{T}$ & & & & & & & \\
\hline \multicolumn{11}{|l|}{ VOLE species } \\
\hline \multirow{4}{*}{$\begin{array}{l}\text { MOUSE species } \\
\text { NORWAY RAT } \\
\text { HOUSE MOUSE } \\
\text { PORCUPINE }\end{array}$} & & $5 \mathrm{~T}$ & & & $5 T$ & & & & 4 & \\
\hline & & $\mathrm{T}$ & & & & & & & & \\
\hline & & & & & & & & & 1 & \\
\hline & 1 & & & 2 & & & 1 & & 1 & \\
\hline \multirow{5}{*}{$\begin{array}{l}\text { COYOTE } \\
\text { WOLF } \\
\text { RED FOX } \\
\text { SWIFT FOX } \\
\text { BLACK BEAR }\end{array}$} & $\mathrm{H}$ & $4 \mathrm{~T}$ & $\mathrm{~T}$ & $1 \mathrm{~T}$ & & $6 \mathrm{~T}$ & 1 & $2 \mathrm{~T}$ & 4 & 2 \\
\hline & & & & & & 1T & & & & \\
\hline & 2 & & $\mathrm{~T}$ & 1 & & $1 \mathrm{~T}$ & & & & 1 \\
\hline & & & & & & & & & & \\
\hline & 1 & & & & & $1 \mathrm{~L}$ & & & & \\
\hline RACOON & 3 & & & & & & & & & \\
\hline \multicolumn{11}{|l|}{ FISHER } \\
\hline \multicolumn{11}{|l|}{ ERMINE } \\
\hline \multirow{2}{*}{$\begin{array}{l}\text { LONG-TAILED WEASEL } \\
\text { LEAST WEASEL }\end{array}$} & & $2 \mathrm{~T}$ & $1 \mathrm{~T}$ & & & & & & & \\
\hline & & & & & & & & & & \\
\hline \multirow{5}{*}{$\begin{array}{l}\text { WEASEL species } \\
\text { MINK } \\
\text { BADGER } \\
\text { STRIPED SKLNK } \\
\text { RIVER OTTER }\end{array}$} & & & & & & $3 \mathrm{~T}$ & & & & \\
\hline & & & & & & $1 \mathrm{~T}$ & & $1 \mathrm{~T}$ & & \\
\hline & & & & & & & & & & \\
\hline & & & & & & & & & & \\
\hline & & & & & & & & & & \\
\hline \multicolumn{11}{|l|}{ MOUNTAIN LION } \\
\hline \multicolumn{11}{|l|}{ WOODLAND CARIBOU } \\
\hline \multirow{2}{*}{$\begin{array}{l}\text { MULE DEER } \\
\text { WHITE-TAILED DEER }\end{array}$} & & & & & & & 4 & & 12 & 26 \\
\hline & 86 & $3+40 \mathrm{~T}$ & 2 & 38 & & 6 & 3 & 22 & 20 & 12 \\
\hline \multicolumn{11}{|l|}{ DEER species } \\
\hline \multirow{3}{*}{$\begin{array}{l}\text { MOOSE } \\
\text { ELK } \\
\text { PRONGHORN } \\
\end{array}$} & 8 & & & & & $2 T$ & & & & \\
\hline & 18 & & & & & $15 \mathrm{~T}$ & & & & \\
\hline & & & & & & & & & & \\
\hline TOTAL SPECIES & 10 & 7 & 8 & 9 & 2 & 12 & 5 & 5 & 8 & \\
\hline
\end{tabular}


Table 1-6: SASKATCHEWAN CHRISTMAS MAMMAL COUNTS

\begin{tabular}{|c|c|c|c|c|c|c|c|c|c|c|}
\hline \multirow[t]{6}{*}{ 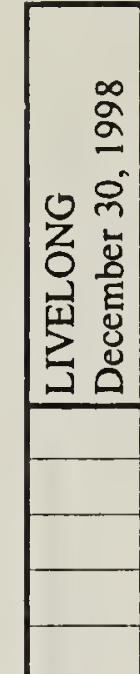 } & 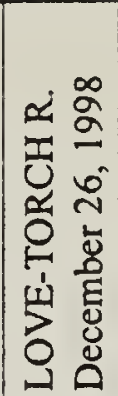 & 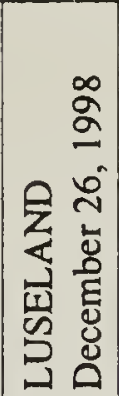 & 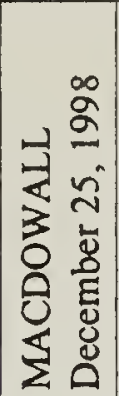 & 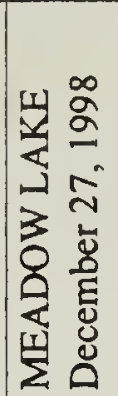 & 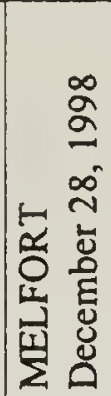 & 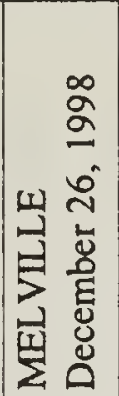 & 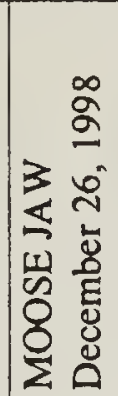 & 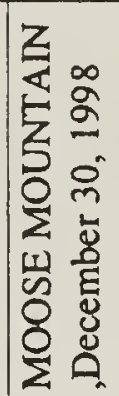 & 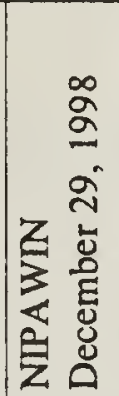 & 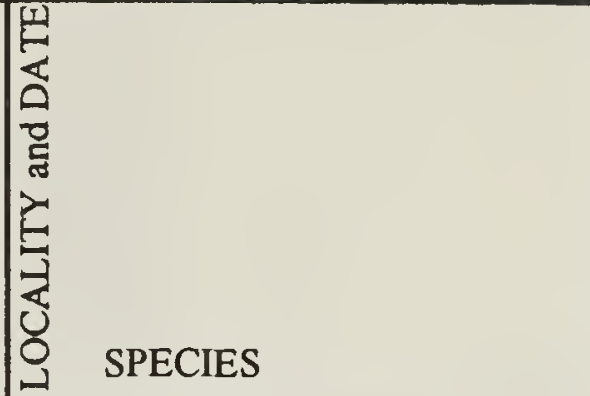 \\
\hline & & & & & & & & & & \multirow{5}{*}{$\begin{array}{l}\text { MASKED SHREW } \\
\text { SHREW species } \\
\text { EASTERN COTTONTAIL } \\
\text { NUTTALL'S COTTONTAIL }\end{array}$} \\
\hline & & & $2 \mathrm{~T}$ & & & & & & & \\
\hline & & & & & & & & & & \\
\hline & & & & & & & & & & \\
\hline & & & $4 \mathrm{~T}$ & $25 \mathrm{~T}$ & & & & & & \\
\hline \multirow[t]{15}{*}{1} & & 1 & $1 \mathrm{~T}$ & & & & 7 & & & \multirow{5}{*}{$\begin{array}{l}\text { WHITE-TAILED JACKRABBIT } \\
\text { GREY SQUIRREL } \\
\text { FOX SQUIRREL } \\
1 \text { RED SQUIRREL } \\
\text { NORTHERN FLYING SQUIRREI }\end{array}$} \\
\hline & & & & & & & & & & \\
\hline & & & & & & & 11 & & & \\
\hline & 4 & & 2 & $2 \mathrm{H}$ & & & & 6 & 1 & \\
\hline & & & & & & & & & & \\
\hline & & & & & & & & & & \multirow{5}{*}{$\begin{array}{l}\text { BEAVER } \\
\text { DEER MOUSE } \\
\text { GAPPER'S RED-BACKED VOLE } \\
\text { MUSKRAT } \\
\text { MEADOW VOLE }\end{array}$} \\
\hline & & & & & & & & & & \\
\hline & & & & & & & & & & \\
\hline & & & & & & & & & & \\
\hline & & & & & & & & & & \\
\hline & & & & & & & & & & VOLE species \\
\hline & & & & $12 \mathrm{~T}$ & & $1 \mathrm{~T}$ & & & & MOUSE species \\
\hline & & & & & & & & & & NORWAY RAT \\
\hline & & & & & & & & & & HOUSE MOUSE \\
\hline & & & IT & & & & & 1 & & PORCUPINE \\
\hline \multirow[t]{2}{*}{2} & & 2 & $1+3 \mathrm{~T}$ & $1 \mathrm{~T}$ & 1 & & & 3 & & COYOTE \\
\hline & & & & & & & & & & WOLF \\
\hline \multirow[t]{6}{*}{+} & & & $2 \mathrm{~T}$ & & & IT & & 1 & & RED FOX \\
\hline & & & & & & & & & & SWIFT FOX \\
\hline & & & & & & & & & & BLACK BEAR \\
\hline & & & $1 \mathrm{~T}$ & & & & & & & RACOON \\
\hline & & & & & & & & & & FISHER \\
\hline & & & & & & & & & & ERMNE \\
\hline \multirow[t]{9}{*}{ t } & & & & & & & & & & \multirow{2}{*}{$\begin{array}{l}\text { LONG-TAILED WEASEL } \\
\text { LEAST WEASEL }\end{array}$} \\
\hline & & & & & & & & & & \\
\hline & & & $2 \mathrm{~T}$ & & & $1 \mathrm{~T}$ & & & & WEASEL species \\
\hline & & & & & & & & & & MINK \\
\hline & & & & & & & & & & BADGER \\
\hline & & & & & & & & & & STRIPED SKUNK \\
\hline & & & & & & & & & & RIVER OTTER \\
\hline & & & & & & & & & & MOUNTAIN LION \\
\hline & & & & & & & & & & WOOF_AND CARIBOU \\
\hline t & & 17 & & & & & & & 7 & MULE DEER \\
\hline \multirow[t]{5}{*}{5} & 15 & 7 & $20 \mathrm{~T}$ & $8+30 \mathrm{~T}$ & 2 & $2 \mathrm{~T}$ & 6 & 18 & & WHITE-TAILED DEER \\
\hline & & & & & & & & & & DEER species \\
\hline & & 2 & & & & & & & & MOOSE \\
\hline & & & & & & & & & & ELK \\
\hline & & & & & & & & & & PRONGHORN \\
\hline 6 & 2 & 5 & 10 & 5 & 2 & 4 & 3 & 5 & 2 & TOTAL SPECIES \\
\hline
\end{tabular}


Table 1-7: SASKATCHEWAN CHRISTMAS MAMMAL COUNTS

\section{SPECIES}

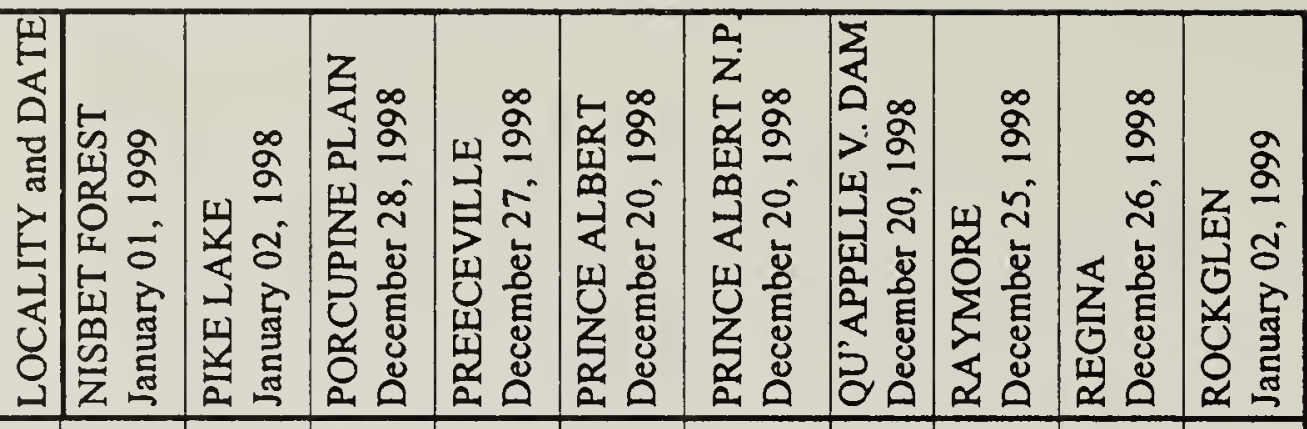

\section{MASKED SHREW}

SHREW species

EASTERN COTTONTAIL

NUTTALL'S COTTONTAIL

SNOWSHOE HARE

WHITE-TAILED

GREY SQUIRREL

FOX SQUIRREL

RED SQUIRREL

NORTHERN FLYING

BEAVER

DEER MOUSE

GAPPER'S RED-BACKED

MUSKRAT

MEADOW VOLE

VOLE species

MOUSE species

NORWAY RAT

HOUSE MOUSE

PORCUPINE

COYOTE

WOLF

RED FOX

SWIFT FOX

BLACK BEAR

RACOON

FISHER

ERMINE

LONG-TAILED WEASEL

LEAST WEASEL

WEASEL species

MINK

BADGER

STRIPED SKUNK

RIVER OTTER

MOUNTAIN LION

WOODLAND CARIBOU

MULE DEER

WHITE-TAILED DEER

DEER species

MOOSE

ELK

PRONGHORN

TOTAL SPECIES

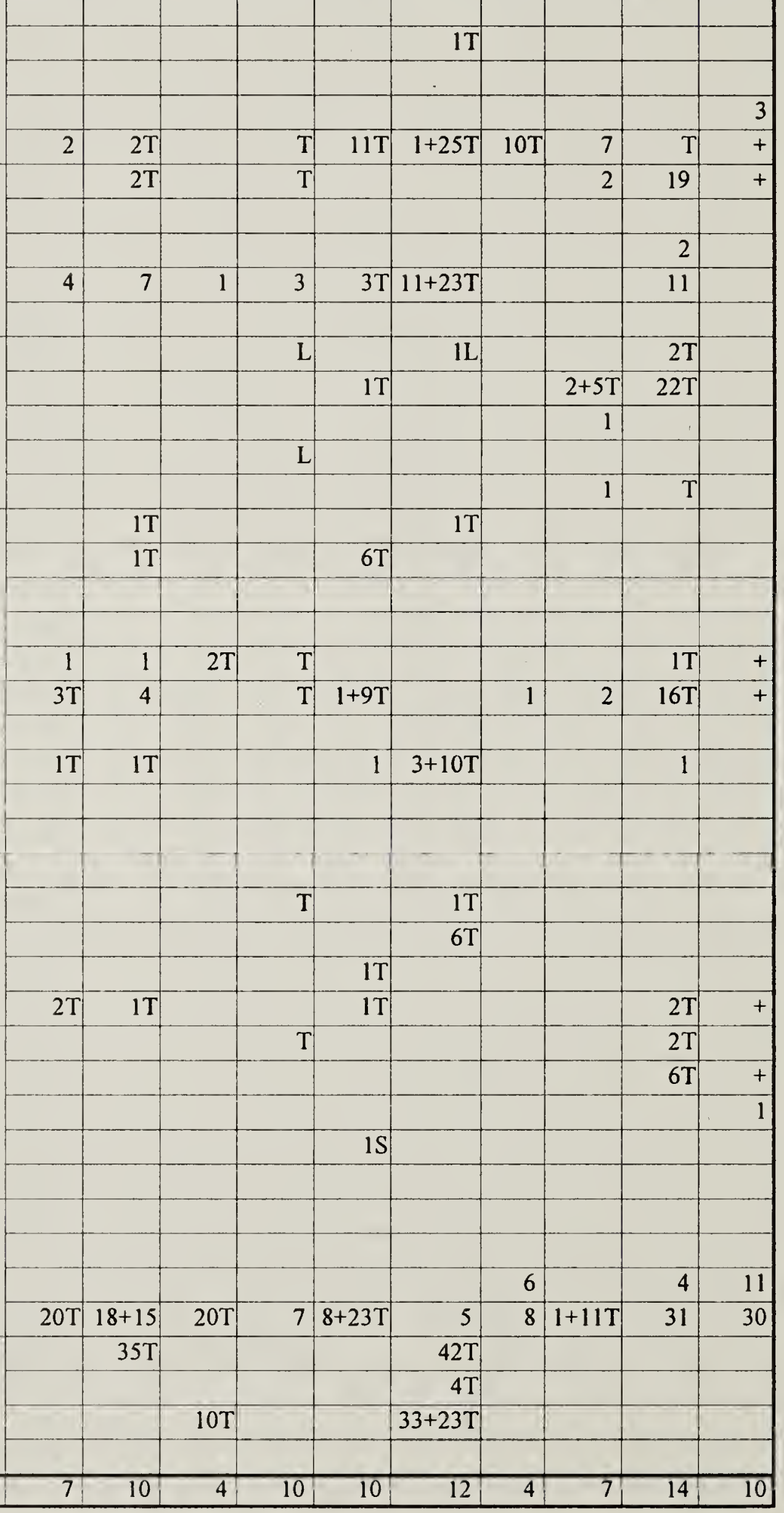


Table 1-8: SASKATCHEWAN CHRISTMAS MAMMAL COUNTS

\begin{tabular}{|c|c|c|c|c|c|c|c|c|c|c|}
\hline 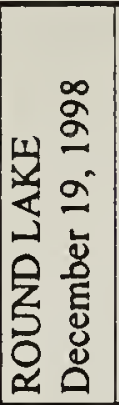 & 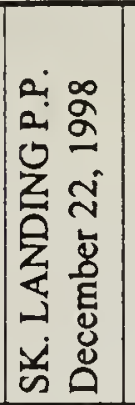 & 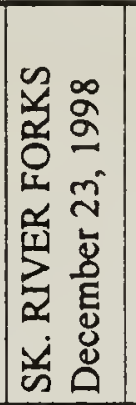 & 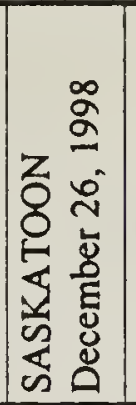 & 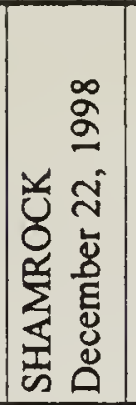 & 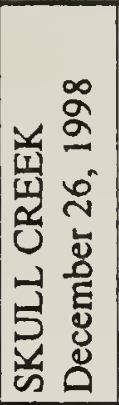 & 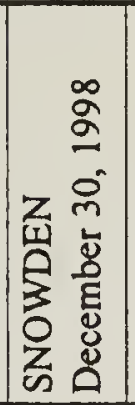 & 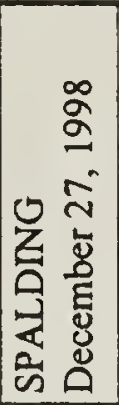 & 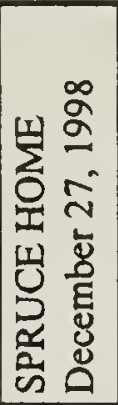 & 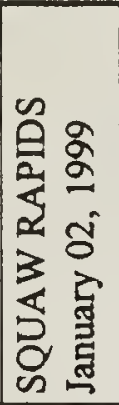 & 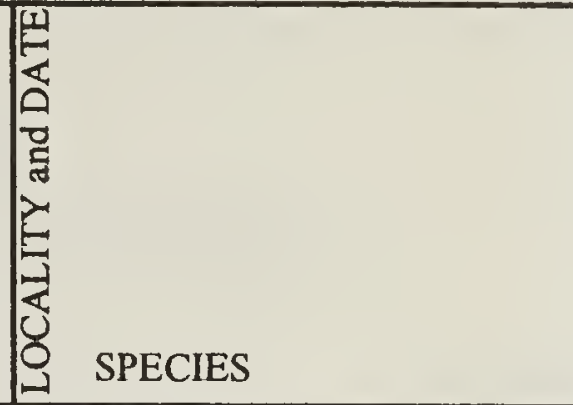 \\
\hline & $1 \mathrm{~T}$ & & & & & & & & & MASKED SHREW \\
\hline & & $1 \mathrm{~T}$ & & & & & $\mathrm{~T}$ & & $1 \mathrm{~T}$ & SHREW species \\
\hline & & & & & & & & & & EASTERN COTTONTAIL \\
\hline & $2+11 \mathrm{~T}$ & & & & & & & & & NUTTALL'S COTTONTAIL \\
\hline $12 \mathrm{~T}$ & & $8 \mathrm{~T}$ & $4+20 \mathrm{~T}$ & $5 \mathrm{~T}$ & & $20 \mathrm{~T}$ & $\mathrm{~T}$ & & $7 \mathrm{~T}$ & SNOWSHOE HARE \\
\hline $2 \mathrm{~T}$ & $2 \mathrm{~T}$ & $1 \mathrm{~T}$ & 3 & & 1 & & & & $1 \mathrm{~T}$ & WHITE-TAILED JACKRABBIT \\
\hline & & & & & & & & & & GREY SQUIRREL \\
\hline & & & & & & & & & & FOX SQUIRREL \\
\hline 7 & & $5+4 \mathrm{~T}$ & $1+1 \mathrm{~T}$ & & & 8 & & & 12 & RED SQUIRREL \\
\hline & & & & & & & & & & NORTHERN FL YING SQUIRREI \\
\hline $1 \mathrm{~L}$ & & & & & & & & & $2 \mathrm{~L}$ & BEAVER \\
\hline & $2 \mathrm{~T}$ & $5 \mathrm{~T}$ & $3 \mathrm{~T}$ & & & & & $1 \mathrm{~T}$ & $2 \mathrm{~T}$ & DEER MOUSE \\
\hline & & & & & & & & & & GAPPER'S RED-BACKED VOLE \\
\hline 1 & & & & & & & & & $7 \mathrm{~L}$ & MUSKRAT \\
\hline $12 \mathrm{~T}$ & & & $1 \mathrm{~T}$ & & & $3 \mathrm{~T}$ & & & $8 \mathrm{~T}$ & MEADOW VOLE \\
\hline & $2 \mathrm{~T}$ & & & & & & $\mathrm{~T}$ & & & VOLE species \\
\hline & & $2 \mathrm{~T}$ & $4+2 T$ & & & $10 \mathrm{~T}$ & $\mathrm{~T}$ & & & MOUSE species \\
\hline & & & & & & & & & & NORWAY RAT \\
\hline & & & & & & & & & & HOUSE MOUSE \\
\hline 1 & 2 & & $2+1 \mathrm{~T}$ & & & + & & & & PORCUPINE \\
\hline 4 & $1+3 \mathrm{~T}$ & $4 \mathrm{~T}$ & $7+6 \mathrm{~T}$ & & 6 & & $\mathrm{~T}$ & & $5 T$ & COYOTE \\
\hline & & & & & & & & & $\overline{2+2 T}$ & WOLF \\
\hline $10 \mathrm{~T}$ & & $1 \mathrm{~T}$ & $1+3 \mathrm{~T}$ & $2 \mathrm{~T}$ & 1 & & $\mathrm{~T}$ & & 1 & RED FOX \\
\hline & & & & & & & & & & SWIFT FOX \\
\hline & & & & & & & & & & BLACK BEAR \\
\hline & & & & & & $2 \mathrm{~T}$ & & & & RACOON \\
\hline & & & & & & & & & & FISHER \\
\hline & & & & & & & & & $2 \mathrm{~T}$ & ERMINE \\
\hline & & $2 \mathrm{~T}$ & & & & $1 \mathrm{~T}$ & & & & LONG-TAILED WEASEL \\
\hline & & & & & 1 & + & & & & LEAST WEASEL \\
\hline & $1 \mathrm{~T}$ & & $2 \mathrm{~T}$ & & & & & & $3 T$ & WEASEL species \\
\hline 2 & 1 & $1 \mathrm{~T}$ & & & & & & & $1 \mathrm{~T}$ & MINK \\
\hline & & & & & & & & & & BADGER \\
\hline & & & & & & & & & & STRIPED SKUNK \\
\hline & & & & & & & & & $1 \mathrm{~T}$ & RIVER OTTER \\
\hline & & & & & & & & & & MOUNTAIN LION \\
\hline & & & & & & & & & & WOODLAND CARIBOU \\
\hline & 38 & & & & 22 & & & & & MULE DEER \\
\hline 3 & 52 & $2+2 \mathrm{~T}$ & 22 & $8+2 T$ & 7 & & $\mathrm{~T}$ & & 5 & WHITE-TAILED DEER \\
\hline & & & $9 \mathrm{~T}$ & & & & & & & DEER species \\
\hline & & & & & & & & & $1 T$ & MOOSE \\
\hline & & & & & & 1 & & & $11+5 T$ & ELK \\
\hline & 28 & & & & & & & & & PRONGHORN \\
\hline 11 & 12 & 10 & 10 & 3 & 6 & 9 & 7 & 1 & 18 & TOTAL SPECIES \\
\hline
\end{tabular}


Table 1-9: SASKATCHEWAN CHRISTMAS MAMMAL COUNTS

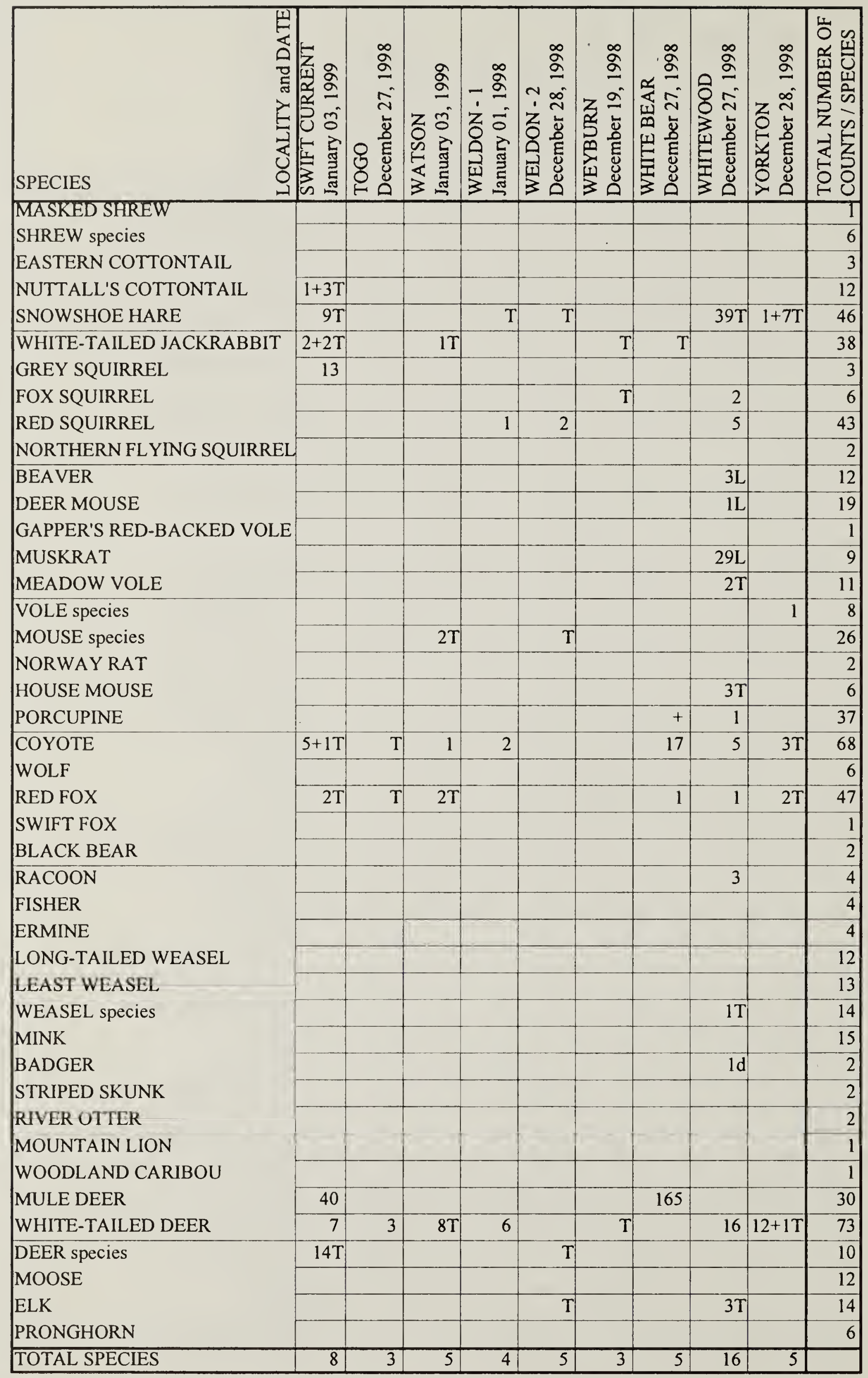

